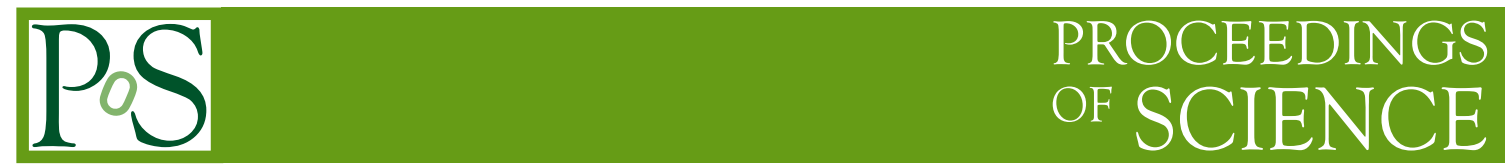

\title{
Muon reconstruction performance in ATLAS at Run-2
}

\section{Hannah Herde* on behalf of the ATLAS Collaboration}

Brandeis University (US)

E-mail: hannah.herdedcern.ch

The ATLAS muon reconstruction has been improved for the Run-2 of the LHC. In this presentation, we will discuss its performance as measured during the early run of the LHC in 2015 at $\sqrt{s}=13 \mathrm{TeV}$ using samples of $J / \psi \rightarrow \mu \mu$ and $Z \rightarrow \mu \mu$ decays. Reconstruction efficiency, transverse momentum resolution and momentum scales are measured in the various regions of the detector and for muon momenta between 5 and hundreds of $\mathrm{GeV}$. They are all found to be in good agreement with tuned Monte Carlo simulations.

The European Physical Society Conference on High Energy Physics 22-29 July 2015

Vienna, Austria

${ }^{*}$ Speaker. 


\section{Introduction}

This note provides a first look at the performance of the ATLAS muon reconstruction in early Run-2 at the LHC. Dimuon resonances $\left(J / \psi \rightarrow \mu^{+} \mu^{-}\right.$and $\left.Z \rightarrow \mu^{+} \mu^{-}\right)$are used to measure muon reconstruction and isolation efficiencies as a function of muon transverse momentum and pseudorapidity. Additionally, Monte Carlo (MC) simulations using GEANT4 with realistic ATLAS geometry, resolution, and alignment allow modeling of interactions within the detector. At the level of necessary precision, small discrepancies from the imperfect description of detector materials, residual misalignments in the Inner Detector (ID) or Muon Spectrometer (MS), and other sources must be corrected by applying smearing and scale corrections to the simulated muon momenta. A preliminary validation of these momentum corrections is also presented.

\section{Detector description and muon reconstruction}

A full description of the ATLAS detector can be found in reference [2]. Muons are primarily identified and reconstructed by combining information from the ID and MS detectors.

The ID is the main tracking detector, operating with acceptance $|\eta|<2.5^{1}$ in a 2 T axial magnetic field. It consists of three sub-detectors: three layers of Silicon Pixels $(5 \mathrm{~cm}<\mathrm{r}<12 \mathrm{~cm}$ ) and four layers of silicon strips $(30 \mathrm{~cm}<\mathrm{r}<51 \mathrm{~cm}$ ) span the full ID acceptance, while 72 straw layers of transition radiation tracker modules $(55 \mathrm{~cm}<\mathrm{r}<108 \mathrm{~cm})$ have a pseudorapidity coverage up to $|\eta|<2.0$.

The MS is the outermost ATLAS sub-detector. This muon tracking detector provides independent momentum measurements of charged particles with a relative resolution better than $3 \%$ over a wide $\mathrm{p}_{\mathrm{T}}$ range and up to $10 \%$ at $\mathrm{p}_{\mathrm{T}} \sim 1 \mathrm{TeV}$. It operates in the pseudorapidity region $|\eta|<2.7$. The MS consists of one barrel $(|\eta|<1.05)$ and two end-cap sections. A system of three large superconducting air-core toroidal magnets provides a magnetic field of $0.5 \mathrm{~T}$. Three layers of resistive plate chambers (RPCs) $(|\eta|<1.05)$ and three layers of thin gap chambers $(1.05<|\eta|<2.4)$ serve as trigger chambers for the detector and provide $(\eta, \phi)$ position measurements with typical spatial resolution of 5-10 mm. Three layers of monitored drift tube (MDTs) chambers $(|\eta|<2.7)$ provide precise muon momentum measurements. Each chamber provides six to eight $\eta$ measurements along the muon track. The innermost layer of MDTs is replaced by cathode strip chambers in $|\eta|>2$.0. During the shutdown preceding Run-2, the last missing chambers in the transition region between the barrel and end-caps $(1.0<|\eta|<1.4)$ were added, completing the MS to its initial design specifications [3]. In addition, four RPC-equipped MDT chambers were installed inside two elevator shafts to improve the efficiency in that region relative to Run-1.

About $96 \%$ of muons are reconstructed by fitting hits from ID and MS tracks, producing combined muons. The remainder are formed by tagging ID tracks with muon signatures in the calorimeter or the MS.

\footnotetext{
${ }^{1}$ ATLAS uses a right-handed coordinate system with its origin at the nominal interaction point (IP) in the centre of the detector and the $z$-axis along the beam pipe. The $x$-axis points from the IP to the centre of the LHC ring, and the $y$-axis points upward. Cylindrical coordinates $(r, \phi)$ are used in the transverse plane, $\phi$ being the azimuthal angle around the beam pipe. The pseudorapidity is defined in terms of the polar angle $\theta$ as $\eta=-\ln \tan (\theta / 2)$.
} 


\section{Muon identification}

Muons are selected for analysis using a set of requirements developed to reject delayed muons coming from pion and kaon decays and to guarantee a reliable momentum measurement. Four muon selections are currently maintained for use in physics analyses: Loose, Medium, Tight, and $H i g h-\mathrm{p}_{\mathrm{T}}$. The Loose identification criteria are designed to maximize muon reconstruction efficiency while ensuring dependable muon tracks. They are specifically optimized for reconstructing Higgs boson candidates in the four-lepton final state [5]. The Medium criteria, representing the default selection for muons in ATLAS, is designed to minimize systematic uncertainties associated with muon reconstruction and calibration. The Tight criteria minimize the fake muon rate to optimize sample purity. Finally, the High-p $\mathrm{p}_{\mathrm{T}}$ selection aims to maximize momentum resolution for tracks with transverse momentum above $100 \mathrm{GeV}$.

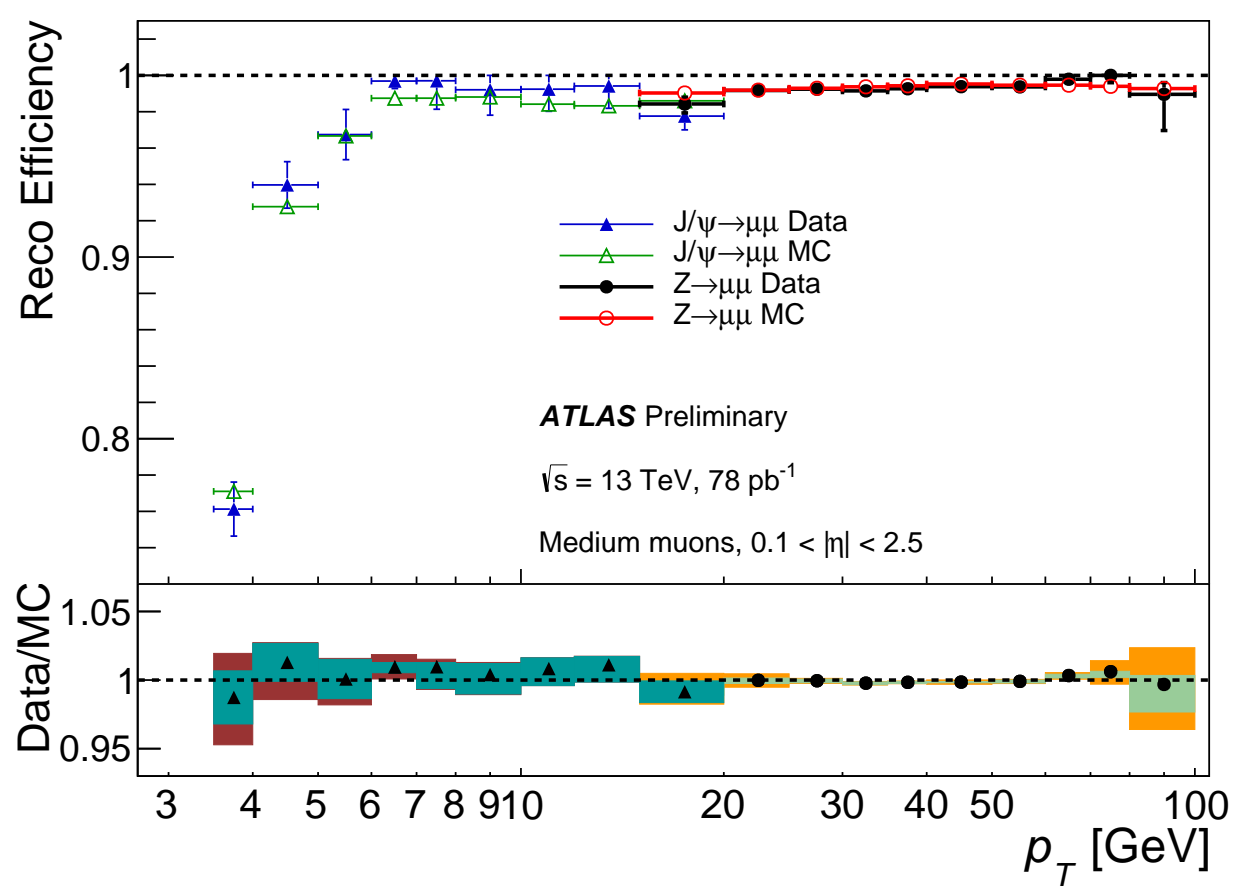

Figure 1: Muon reconstruction efficiency for the Medium identification algorithm measured in $J / \psi \rightarrow$ $\mu^{+} \mu^{-}$and $Z \rightarrow \mu^{+} \mu^{-}$events as a function of the muon momentum. The prediction by the detector simulation is depicted as red circles (green triangles), while black dots (blue triangles) indicate the observation in collision data for $Z \rightarrow \mu \mu(J / \psi \rightarrow \mu \mu)$ events. The bottom panel reports the the efficiency scale factors. Green (cyan) error bands indicate the statistical uncertainty, and orange (red) bands the quadratic sum of statistical and systematic uncertainty for $Z \rightarrow \mu \mu(J / \psi \rightarrow \mu \mu)$ events. In one bin $\left(15 \mathrm{GeV}<\mathrm{p}_{\mathrm{T}}<20 \mathrm{GeV}\right)$, results are given for both analyses. The luminosity indicated in the figure refers to the period in which all triggers for muon reconstruction efficiency were active for both resonances. Taken from Ref. [6].

\section{Muon reconstruction and isolation}

Muon reconstruction and isolation efficiencies are measured using a Tag and Probe method [4] 
applied to $Z \rightarrow \mu^{+} \mu^{-}$events. Reconstruction efficiency measurements also benefit from $J / \psi \rightarrow$ $\mu^{+} \mu^{-}$decays for a sample of low transverse momentum muons $\left(2.5 \mathrm{GeV}<\mathrm{p}_{\mathrm{T}}<15 \mathrm{GeV}\right) . Z \rightarrow$ $\mu^{+} \mu^{-}$decays are selected by requiring the tag and probe muons to have opposite charge and an invariant mass with $10 \mathrm{GeV}$ of the $Z$ boson mass, and the tag muon must be isolated.

\subsection{Muon reconstruction efficiencies}

The tag muon is required to be a combined muon which triggered the event readout. The probe must have a track reconstructed in the ID. The fraction of signal probes reconstructed as muons by successfully combining with an MS track measures the muon identification efficiency. A probe is considered successfully reconstructed if a reconstructed muon is found within a cone in the $\eta-\phi$ plane of size $\Delta R=\sqrt{\Delta \eta^{2}+\Delta \phi^{2}}<0.05$ around the probe track. Muon reconstruction efficiencies are extracted separately for MC and data as a function of pseudorapidity and probe transverse momentum. High efficiencies, close to $99 \%$, are observed across all detector regions except the crack region $(|\eta|<0.1)$. In this region, the efficiency is $<60 \%$ on account of gaps between muon chambers for ID and calorimeter services. These results are in good agreement with the detector simulation prediction. The reconstruction efficiency as a function of $\mathrm{p}_{\mathrm{T}}$ is shown for Medium muons in figure 1. The plot displays the high reconstruction efficiency across a broad $\mathrm{p}_{\mathrm{T}}$ spectrum ( $>97 \%$ for muons with $7 \mathrm{GeV}<\mathrm{p}_{\mathrm{T}}<100 \mathrm{GeV}$ ), and the good agreement between data and MC.

\subsection{Muon isolation efficiencies}
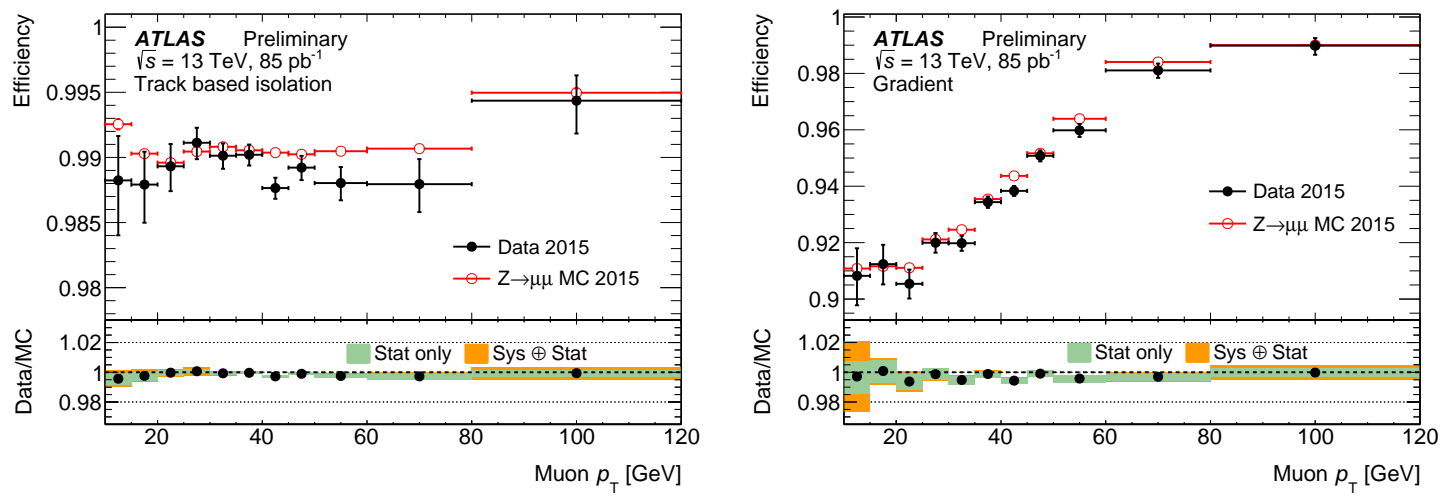

Figure 2: Efficiencies of the track-based isolation as a function of the muon $\mathrm{p}_{\mathrm{T}}$ (left) and of the combined track-based and calorimeter-based isolation as a function of the muon $\mathrm{p}_{\mathrm{T}}$ (right). Muons from $Z \rightarrow \mu^{+} \mu^{-}$ decays are required to pass Medium selection criteria. The last bin includes overflows. Black dots indicate the efficiency measured in data while red circles indicate the prediction from simulation. The errors on the efficiencies are statistical. The bottom panel shows the ratio between data and simulation, as well as the statistical uncertainties (green) and combination of statistical and systematic uncertainties (orange). The largest contribution arises from the mass window cut in the low $\mathrm{p}_{\mathrm{T}}$ region, which suffers from more background, whereas the high $\mathrm{p}_{\mathrm{T}}$ region is dominated by statistical uncertainties and systematics due to the requirement on $\Delta R$ between the two muons. Taken from Ref. [6].

Muon isolation is both track-based and calorimeter-based. The track-based isolation variable is defined as the sum of the transverse momenta of the tracks in a cone around the muon, excluding 
the muon-track itself. The cone size is $\mathrm{p}_{\mathrm{T}}$-dependent to improve identification of muons from boosted particle decays. The calorimeter-based isolation variable is defined as the sum of the energies of the topological clusters around the muon in a cone of radius $\Delta R=0.2$. Clusters within $\Delta R=0.1$ are excluded from the sum, as they may be part of the energy deposit of the muon itself from its passage through the calorimeter. Final isolation cuts used in physics analyses are applied relative to the transverse momentum of the muon for both track- and calorimeter-based isolation. Figure 2 shows efficiency as a function of muon $\mathrm{p}_{\mathrm{T}}$ using a track-based isolation variable (left) and both track- and calorimeter-based isolation variables (right). Again, the data and MC are in good agreement.

\section{Muon momentum scale and resolution}
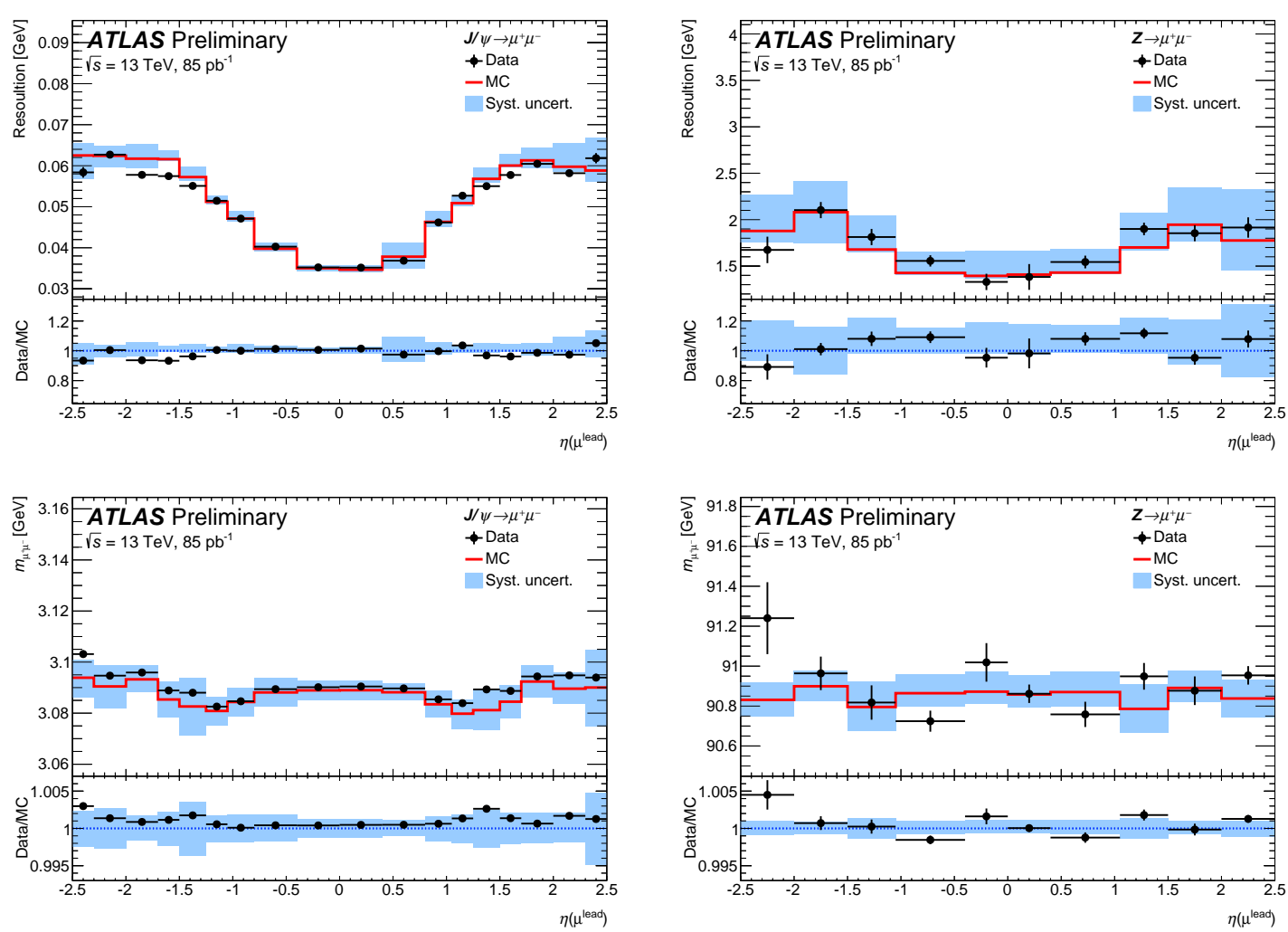

Figure 3: Resolution (top) and mean value (bottom) of the dimuon invariant mass resonance measured for $J / \psi \rightarrow \mu^{+} \mu^{-}$(left) and $Z \rightarrow \mu^{+} \mu^{-}$(right) decays as a function of the pseudorapidity $(\eta)$ of the leading muon. Data (black markers) are compared to simulations (red line). The filled area corresponds to the quadratic sum of all systematic uncertainties. Taken from Ref. [6].

Muon transverse momenta must be calibrated in simulation to provide an accurate description of the momentum resolution and scales. Corrections to the momentum resolution account for multiple scattering, local magnetic field distortions, energy loss due to fluctuations in the traversed material, intrinsic resolution effects, and residual misalignments of the detector. Corrections to the 
scale consider the offset of average energy loss in the calorimeter and other materials, and for radial distortions and mis-modeling of the magnetic field integral.

The $\mathrm{p}_{\mathrm{T}}$ calibration corrections are extracted by fitting the $Z$ and $J / \psi$ invariant mass peaks using a combination of $8 \mathrm{TeV}$ and $13 \mathrm{TeV}$ data. Shown in figure 3 is the test of the validity of the parameterization in $13 \mathrm{TeV}$ data and $\mathrm{MC}$. The plots show the agreement between data and $\mathrm{MC}$ for the mean is within $0.1 \%$ in the barrel region $(|\eta|<1.05)$ and $0.2 \%$ in the end caps $(1.05<|\eta|<$ 2.5). The resolution measured in data agrees with MC to within 3\%. Limited statistics prevent a full calibration at this time.

\section{Conclusion}

The initial performance of the ATLAS muon reconstruction in Run-2 reveals both the reconstruction and isolation efficiencies are high ( $>99 \%$ in most detector regions) and in good agreement with simulation. The muon momentum resolution and scales are also found to be in good agreement with the corrected MC simulation.

\section{References}

[1] ATLAS Collaboration, Performance of the ATLAS Trigger System in 2010, Eur. Phys. J. C 72 (2012) 1849, arXiv: 1110.1530 [hep-ex].

[2] ATLAS Collaboration, The ATLAS Experiment at the CERN Large Hadron Collider, JINST 3 (2008) S08003.

[3] ATLAS Collaboration, ATLAS muon spectrometer: Technical design report, CERN-LHCC-97-22, ATLAS-TDR-10 (1997).

[4] ATLAS Collaboration, Measurement of the muon reconstruction performance of the ATLAS detector using 2011 and 2012 LHC proton-proton collision data, Eur. Phys. J. C74.11 (2014) 3130, arXiv: 1407.3935 [hep-ex].

[5] ATLAS Collaboration, Measurements of Higgs boson production and couplings in the four-lepton channel in pp collisions at center-of-mass energies of 7 and $8 \mathrm{TeV}$ with the ATLAS detector, Phys. Rev. D91.1 (2015) 012006, arXiv: 1408.5191 [hep-ex].

[6] . ATLAS Collaboration, Muon reconstruction performance in early $\sqrt{s}=13 \mathrm{TeV}$ data, ATL-PHYS-PUB-2015-037 (2015). 